\title{
Chaos in a Single Op-Amp-Based Jerk Circuit: Experiments and Simulations
}

\author{
Robert Tchitnga, Tekou Nguazon, Patrick H. Louodop Fotso, and Jason A. C. Gallas
}

\begin{abstract}
We report a five-component autonomous chaotic oscillator of jerky type, hitherto the simplest of its kind, using only one operational amplifier. The key component of the circuit is a junction field-effect transistor operating in its triode region, which provides a nonlinear resistor of antisymmetrical current-voltage characteristic, emulating a Colpitts-like chaotic circuit. We describe the experimental results illustrating the dynamical behavior of the circuit. In addition, we report numerical simulations of a model of the circuit which display good agreement with our measurements.
\end{abstract}

Index Terms-Bifurcations, jerk oscillator, junction field-effect transistor (JFET), piecewise (PW) nonlinearity (NLT), simplest chaotic circuit.

\section{INTRODUCTION}

$\mathbf{T}$ HE quest for the "simplest" system of ordinary differential equations for chaos [1]-[5], the "simplest" chaotic jerk function [5]-[10], and the "simplest" chaotic nonautonomous [11]-[14] as well as autonomous circuits [15]-[26] has been keeping several workers busy during the last four decades. In the meantime, records have been established or beaten, so that Piper and Sprott [27] believe that there has not yet been a decisive winner. To avoid misunderstanding the notion of simplicity, these authors proposed three forms of it: 1) the mathematical simplicity, for which the equations with less terms and less complicated nonlinearities (NLTs) are considered and can be numerically simulated; 2) the simplicity in the circuitry, for which a simple circuit should minimize both the number of physical components and that of idealized elements employed in the model; and, finally, 3) the simplicity from a practical

Manuscript received June 25, 2015; revised September 2, 2015; accepted September 20, 2015. Date of publication September 28, 2015; date of current version February 25, 2016. The work of P. H. Louodop Fotso was supported by São Paulo Research Foundation (FAPESP) under Grant 2014/13272-1. The work of J. A. C. Gallas was supported by CNPq, Brazil. This brief was recommended by Associate Editor T. S. Gotarredona.

R. Tchitnga and T. Nguazon are with Laboratory of Electronics and Signal Processing and also the Research Group on Experimental and Applied Physics for Sustainable Development, Department of Physics, Faculty of Science, University of Dschang, Dschang, Cameroon (e-mail: robert.tchitnga@eaphysud. org; tekgua2@yahoo.fr).

P. H. Louodop Fotso is with Laboratory of Electronics and Signal Processing and also the Research Group on Experimental and Applied Physics for Sustainable Development, Department of Physics, Faculty of Science, University of Dschang, Dschang, Cameroon, and also with Instituto de Física TeóricaUNESP, Universidade Estadual Paulista, 01140-070 São Paulo-SP, Brazil (e-mail: louodop.patrick@eaphysud.org).

J. A. C. Gallas is with Instituto de Altos Estudos da Paraíba, 58039-150 João Pessoa-PR, Brazil; with the Department of Physics, Universidade Federal da Paraíba, 58051-970 João Pessoa-PR, Brazil; and also with Institute for Multiscale Simulation, Friedrich-Alexander Universität Erlangen-Nürnberg, 91052 Erlangen, Germany (e-mail: jason.gallas@gmail.com).

Color versions of one or more of the figures in this brief are available online at http://ieeexplore.ieee.org.

Digital Object Identifier 10.1109/TCSII.2015.2483218 standpoint, where robust stand-alone like autonomous circuits can be constructed using the most common components [27]. The aforementioned criteria for the "simplest" chaotic circuit do not include the one mentioned in [17], which is related to the number of locally active elements in the considered circuit.

It appears that it is generally difficult to reconcile the first and second forms of simplicity. The commonly observed tendency is that very simple circuits are usually described with relative complex equations and vice versa [29]. In the longterm challenge to find the simplest jerk function and its corresponding simplest circuit, the authors of [27] have succeeded to an impressive extent. Although jerk functions may offer simpler notations, when they do exist for given circuits [6], [27], certain NLTs found in the simplest systems that can be studied mathematically are rather very difficult (or even impossible) to be implemented physically, like the cubic NLTs [30], compared to the piecewise (PW) ones [9], yet one particular result in the literature review has highly captivated our attention, namely

$$
\dot{x}=y, \quad \dot{y}=z, \quad \dot{z}=-m z \pm y^{2}-x \text {. }
$$

This jerk system is Sprott's answer to the first simplicity criterion mentioned previously [9]. It stands also as the simplest of its class, with just five terms among which one quadratic NLT and one parameter $m$ are for chaos [8]. This record still stands, as it has been confirmed by rigorous demonstrations from $\mathrm{Fu}$ and Heidel [31], that no simpler case can exist.

Now, for the simplest circuits, let us consider the challenge solely for autonomous ones. In this case, circuits from five up to two components have already been found [15]-[21]. However, essentially all of them require idealized components like operational amplifiers (Op-Amps), except for one of them [20]. In electrical engineering, nonidealized and common analog electronic elements are known to be solely resistors, capacitors, inductors, diodes, transistors, and transformers. The long-time predicted and recently discovered memristor [32], [33] belongs naturally also to this category. However, in the literature [17]-[19], it is realized experimentally through analog modeling of its mathematical equations, making it an idealized single component at the actual standpoint of the technology. Although physical ideal [34] memristors [35], [36] and nonideal [34] memristors [37]-[39] were shown to exist, there still does not exist a circuit that is capable of generating chaos using physical memristors.

The present letter which deals with an oscillator belonging to the category of the so-called simple autonomous chaotic circuits was suggested by a remark from Pham et al. concerning a recent article by some of us [20] in the following words [19]: ". . . the authors have named it the simplest chaotic two-component circuit. However, it is notable that, when the 


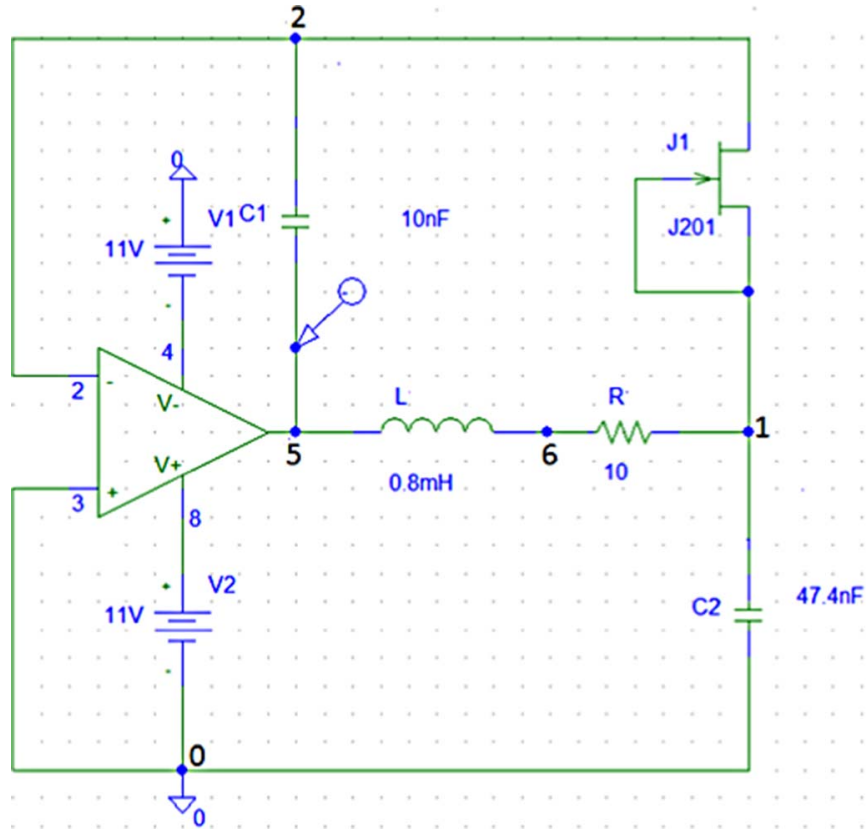

Fig. 1. Schematic representation of the jerky oscillator. The nonlinear element is made of a JFET with the gate $(\mathrm{G})$ and the source $(\mathrm{S})$ electrodes short-circuited.

Tchitnga's circuit is analyzed in terms of the concept of mathematical simplicity ..., it is not really simple because of its four state equations."

Here, we introduce a robust stand-alone five-component circuit of jerky type using a single Op-Amp as a unique idealized element. Its dynamics is described by three state equations having six terms, the same number as that by Piper and Sprott before the simplifying condition to reduce their number to five [27]. Moreover, two from our six terms are made of the same PW nonlinear (NL) term. Before proceeding, we wish to point out two practical purposes for pursuing the simplest possible chaos-generating circuit. First is the didactic purpose of performing simple laboratory experiments, using affordable components, for students learning about chaos. Second, there is a significant theoretical interest in studying the control parameter space of such circuits because they often contain unexpected new structures like periodicity hubs and their associated infinite spirals of stability [28] which induce wide domains of regularity in control space. Currently, there is no theoretical framework to anticipate such structures.

\section{NEW JERK CIRCUIT AND ITS MOdEL}

Consider the phase variable $F=[x, y, z]^{T}$. The nondimensional dynamical flow

$$
\frac{d F}{d t}=\left[\begin{array}{ccc}
0 & 0 & 0 \\
0 & 0 & -\alpha \\
-\beta & \beta & -\beta \gamma
\end{array}\right] F+\left[\begin{array}{l}
1 \\
\alpha \\
0
\end{array}\right] \varphi(y)
$$

with three parameters $\alpha, \beta$, and $\gamma$ and the PW NL term

$$
\varphi(y)=\left\{\begin{array}{lll}
-y & \text { if } \quad y \leq 1 \\
-1 & \text { if } \quad y>1
\end{array}\right.
$$

These equations govern the circuit depicted by Fig. 1: an oscillator with one Op-Amp, one junction field-effect transistor
(JFET), two capacitors $C_{1}$ and $C_{2}$, and one coil $L$ with internal resistance $R$.

Let us consider $R_{J} \approx 750 \Omega$ to be the JFET small signal resistance and $V_{T} \approx-0.66 \mathrm{~V}$ to be the JFET gate-source voltage [40]. An appropriate choice of parameters, namely, $\alpha=C_{1} /$ $C_{2}, \beta=R_{J}^{2} C_{1} / L$, and $\gamma=R / R_{J}$, as well as $I=V_{T} \varphi(y) / R_{J}$, $V_{C_{1}}=x V_{T}, V_{C_{2}}=y V_{T}, I_{L}=z V_{T} / R_{J}$, and $t=R_{J} C_{1} \tau$, reveals the state equations of the circuit, obtained through application of Kirchhoff's laws

$$
\begin{aligned}
C_{1} \dot{V}_{C_{1}} & =I \\
C_{2} \dot{V}_{C_{2}} & =I-I_{L} \\
L \dot{I}_{L} & =-\left(V_{C_{1}}-V_{C_{2}}+R I_{L}\right) .
\end{aligned}
$$

The necessary NLT in the system for chaos is made of the PW linear form of the JFET current-voltage characteristic

$$
I=\frac{1}{R_{J}} \begin{cases}-V_{C_{k}} & \text { if }-V_{C_{k}} \geq V_{T} \\ V_{T} & \text { if }-V_{C_{k}}<V_{T} .\end{cases}
$$

$V_{C_{k}}, k=1,2$, is the voltage difference at the electrodes of capacitor $C_{k}$, and $I_{L}$ is the current flowing through the coil $L$.

With some mathematical manipulations, it can be shown that (2) can be transformed into the jerk form

$$
\dddot{y}=-\alpha\left[\frac{\beta \gamma}{\alpha} \ddot{y}-\ddot{\varphi}(y)+\beta \gamma \dot{\varphi}(y)-\beta \varphi(y)\right] .
$$

Put in its PW linear form as

$$
\dddot{y}=- \begin{cases}(\alpha+\beta \gamma) \ddot{y}+\alpha \beta(1+\gamma) \dot{y}+\alpha \beta y & \text { if } y \leq 1 \\ \beta \gamma \ddot{y}+\alpha \beta \dot{y}+\alpha \beta & \text { if } y>1\end{cases}
$$

Equation (7) can be used to perform exact analytical works. Surprisingly, apart some coefficients, (6) is comparable to

$$
\dddot{x}=-\mu\left[\frac{1}{\mu} \ddot{x}+\ddot{h}(x)(a-b) \dot{x}+\dot{h}(x)+b h(x)\right]
$$

considered by Sprott and Linz [9] as the jerk form modeling Chua's circuit with $h(x)$ as its NL function. However, while in Chua's circuit this three-segment PW NL function is implemented using eight components, here it is realized with just one simple component.

It is worth mentioning that the NLT described by (3) can be replaced by a diode function type known as the min-function, if defined using the reference value " 1 " as

$$
\varphi(y)=-\min (y, 1) .
$$

The single graphical representation for both NLTs (see Fig. 2) confirms our observation.

\section{EQUILIBRIUM POINTS AND BIFURCATIONS}

The equilibrium points are solutions of the equation

$$
\dot{F}=0 \text {. }
$$

Calling $\left(x^{*}, y^{*}, z^{*}\right)$ as the solutions of (10), according to (3), two distinct possibilities do exist.

If $y>1$, then, according to the first relations in (3) and (10), respectively, $\varphi\left(y^{*}\right)=-1=0$, which is impossible. Thus, for $y>1$, there is no equilibrium point.

On the other hand, if $y \leq 1$, then $\varphi\left(y^{*}\right)=-y^{*}=0$, and the equilibrium point is therefore $P=\left(x^{*}=0, y^{*}=0, z^{*}=0\right)$. 


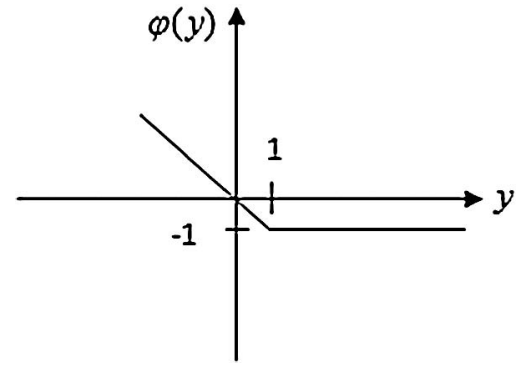

Fig. 2. Common graphical representation of the NLT $\varphi(y)$ both in its PW form like in (3) and as min-function (9). Its implementation involves a single JFET. A representation using a five-component circuit, among which one Op-Amp, is presented by Hudgins et al. [41] citing [42]. A Zener diode could also play such a role, provided that the dimensionless current flowing through it behaves like the Zener voltage in such a way that the avalanche effect starts at the corresponding reference value " 1 ."
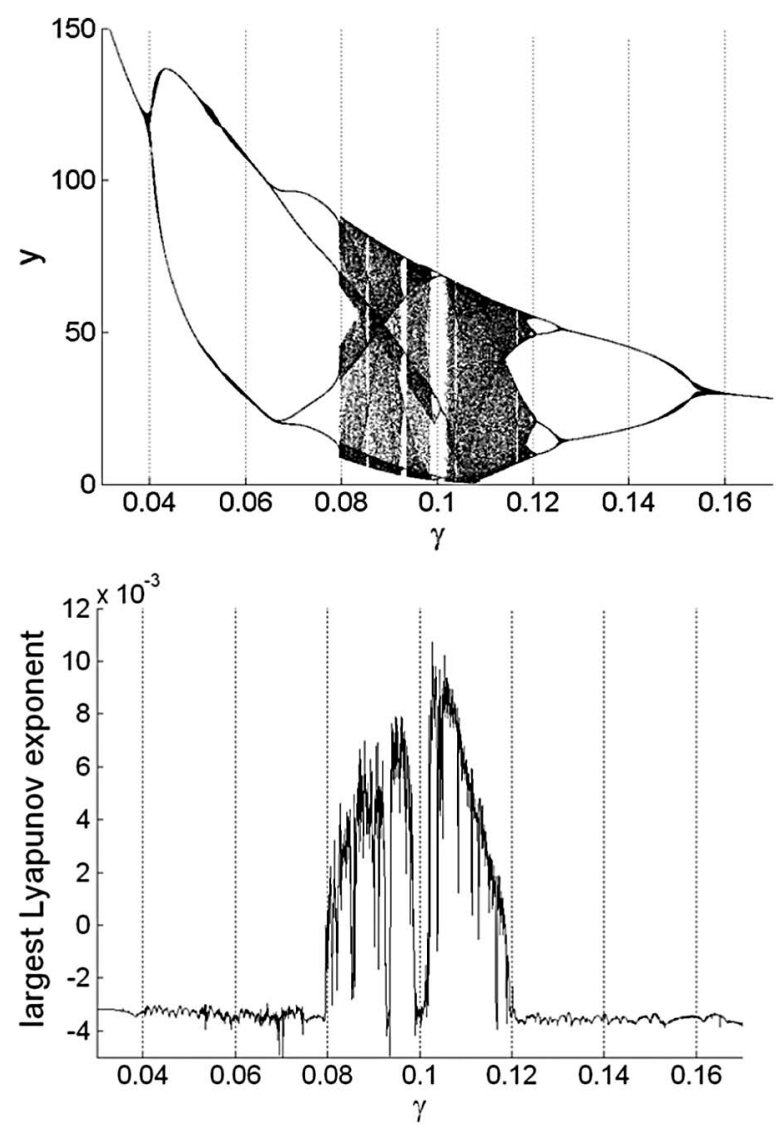

Fig. 3. (a) Bifurcation diagrams displaying the maximum value of $y$ as a function of $\gamma$. (b) Corresponding Lyapunov exponent for $x(0)=0.1, y(0)=$ $-5 \times 10^{-7}, z(0)=-1, \alpha=0.025$, and $\beta=0.765$.

The corresponding Jacobian matrix is

$$
M=\left[\begin{array}{ccc}
0 & -1 & 0 \\
0 & -\alpha & -\alpha \\
-\beta & \beta & -\beta \gamma
\end{array}\right] .
$$

Its eigenvalues are solutions of the characteristic polynomial (in $\lambda$ ) of the linearization at $P$, namely

$$
\lambda^{3}+(\alpha+\beta \gamma) \lambda^{2}+\alpha \beta(1+\gamma) \lambda+\alpha \beta=0 .
$$

If we consider the solution of (12) to be of the form of $\lambda=I \omega$, where $I^{2}=-1$ and $\omega$ is the natural frequency of the system,

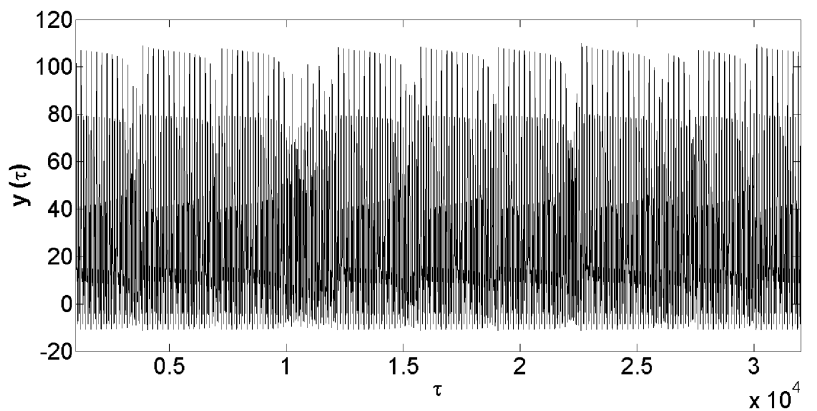

Fig. 4. Intermittency observed in the time evolution of $y$ when $\alpha=0.015 \tau_{0}$, $\beta=0.3 \tau_{0}$, and $\gamma=0.06853 \tau_{0}$.
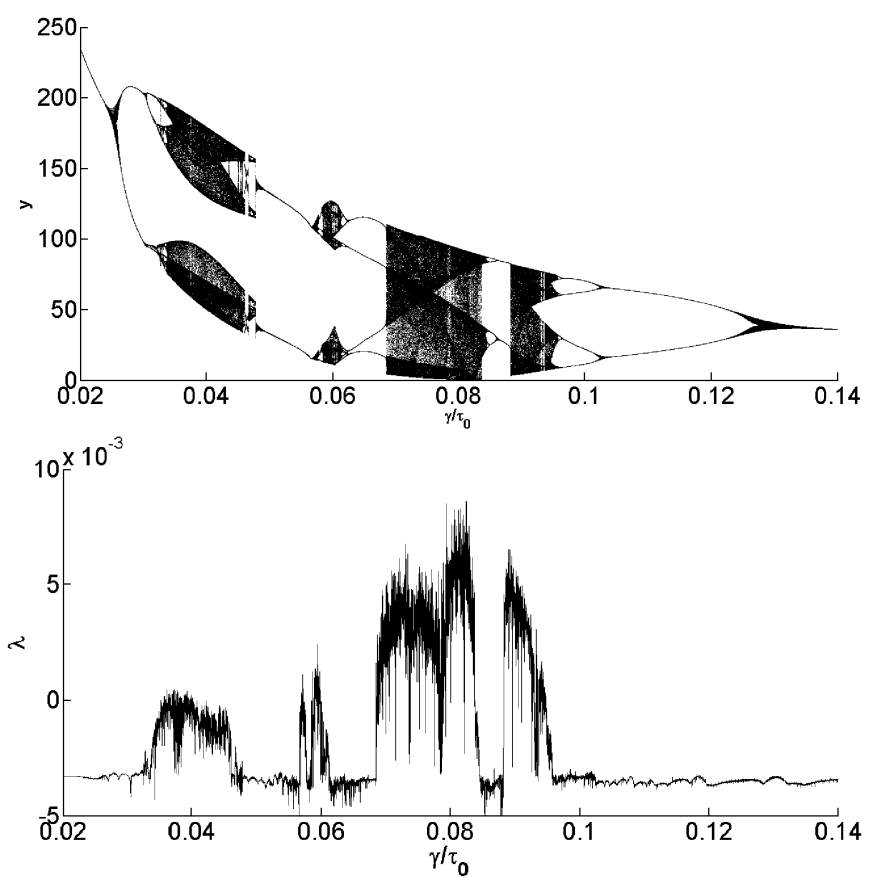

Fig. 5. (a) Bifurcation diagrams resulting from the plot of the maximum value of $y$ when $\gamma / \tau_{0}$ varies. (b) Corresponding Lyapunov exponent for the same initial conditions as in Fig. 3 for $\alpha=0.015 \tau_{0}$ and $\beta=0.3 \tau_{0}$. Here, abrupt crossings from chaos to periodic motions are remarkably clear.

then the following conditions for self-starting are obtained:

$$
\begin{aligned}
\omega & =\sqrt{\frac{\alpha \beta}{\alpha+\beta \gamma}} \\
C_{1}\left(R+R_{J}\right)\left(L+R R_{J} C_{2}\right) & =L C_{2} R_{J} .
\end{aligned}
$$

Using the general form of the solutions to (12), namely $\lambda=$ $a+I b$, and solving them, we obtain the following system of equations as solutions:

$$
\begin{aligned}
a^{3}+(\alpha+\beta \gamma) a^{2}+\alpha \beta(1+\gamma) a+\alpha \beta & =(3 a+\alpha+\beta \gamma) b^{2} \\
\left(3 a^{2}+2(\alpha+\beta \gamma) a-b^{2}+\alpha \beta\right) b & =0
\end{aligned}
$$

For $b=0$, the solution, e.g., $\lambda_{0}$, is real, while for $b \neq 0$, a complex pole-pair solution exists, e.g., $\lambda_{1,2}$. For $\alpha=0.025$, $\beta=0.765$, and $\gamma=0.0875$, corresponding to chaos in the system, the three solutions are found to be $\lambda_{0}=-0.2727557$, situated at the left half $s$-plane (LHP) and $\lambda_{1,2}=0.09040914 \pm$ $24888522 I$ on the RHP. This shows that the oscillator is 

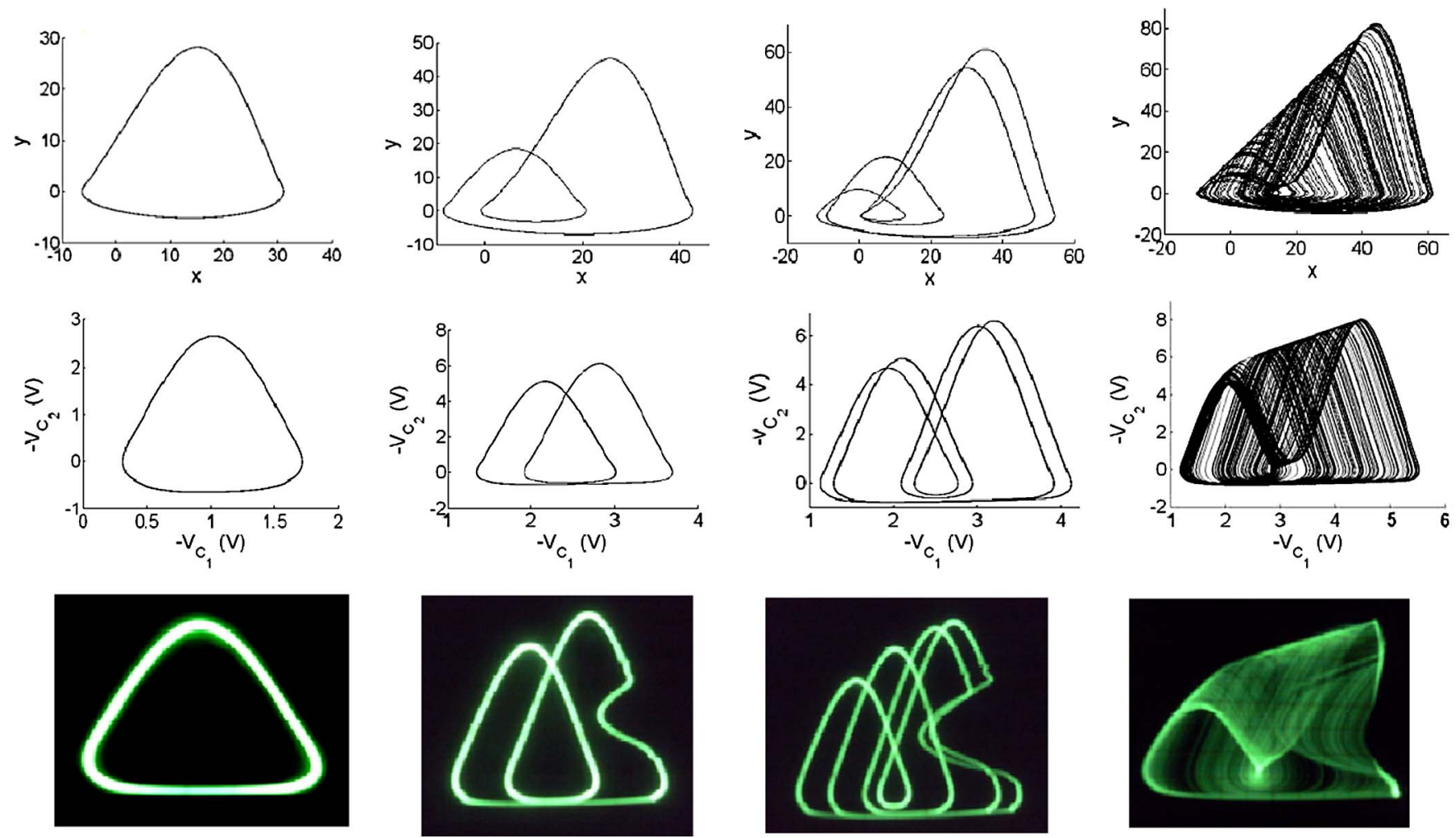

Fig. 6. Comparisons of peak-doubling transition to chaos in the jerk circuit. (Top row) MATLAB-simulated attractors displaying successive peak values and chaos according to the parameter values in the text. (Middle row) PSpice attractors displaying the correspondences to the top row. (Bottom row) Experimental counterpart attractors measured at $X: 0.5 \mathrm{~V} /$ div and $Y: 1 \mathrm{~V} /$ div.

self-starting. The equilibrium point $P$ is unstable and corresponds to a spiral saddle index 2 [43].

If two of the three possible control parameters of the system are maintained constant, e.g., at $\alpha=0.025$ and $\beta=0.765$, then by varying $\gamma$, the third parameter, the system displays various bifurcation states on its route to chaos. While the period varies continuously, we observe a peak-doubling scenario leading to chaos. More explicitly, one peak, two peaks, and four peaks are observed for $\gamma=0.1705, \gamma=0.14$, and $\gamma=0.124$ before the system falls into chaos for $\gamma=0.085$. The bifurcation diagram in Fig. 3(a) illustrates this unfolding for the $y$ variable. This scenario is corroborated by plotting the largest Lyapunov exponent as shown in Fig. 3(b).

Intermittencies in the time domain, which can be seen as frequency packages distinguishable in Fig. 4, are also observed for the example of the variable $y$, as well as interior crisis in Fig. 5, which are abrupt crossings from chaos to periodic motions and vice versa.

To make it more visible, we have induced a proportional expansion of the abscissa axis using an arbitrarily chosen coefficient, the golden number $\tau_{0}=(1 / 2)(1+\sqrt{5})$, to multiply the control parameter. In a mathematical analysis of intermittency in the behavior of major indices near an economic crash, this same technique has previously been used by Ferraro et al. [44] to render the temporal steps discrete using an index evolution.

\section{EXPERIMENTAL EVIDENCE}

The numerical simulation of (2) produces the attractors shown at the top row of Fig. 6, obtained for $\alpha=0.025$ and $\beta=0.765$, and $x(0)=0.1, y(0)=-5 \times 10^{-7}$, and $z(0)=$ -1 . Here, an additional evidence of the peak-doubling route to chaos is confirmed through one-peak, two-peak, and four-peak oscillations and chaos (first row) for $\gamma=0.1705, \gamma=0.14$, $\gamma=0.124$, and $\gamma=0.085$, respectively.

For comparison, a PSpice schematic using an Op-Amp of type TL072/301/TI biased at $\pm 11 \mathrm{~V}$ and a JFET J201 has revealed that, for $C_{1}=10 \mathrm{nF}, C_{2}=47.4 \mathrm{nF}$, and $(L, R)=$ $(0.8 \mathrm{mH}, 10 \Omega)$, the circuit oscillates chaotically. The peakdoubling evolution to chaos is shown at the middle row of Fig. 6 and corresponds to $R=35 \Omega$ for one peak, $R=21 \Omega$ for two peaks, $R=18.5 \Omega$ for four peaks, and $R=10 \Omega$ for chaos.

The laboratory experimental setup was done using the same component types and values as those with PSpice, except for the coil whose physical characteristics were $(0.8 \mathrm{mH}$ and $0.2 \Omega)$ for chaos. An external potentiometer had to be inserted in series with the coil to serve as control parameter. Chaos was still observed when the total serial resistance was in the range $0.2 \Omega \leq$ $R \leq 1.2 \Omega$. However, for periodic motions, higher values of $R$ were needed. Thus, the total value $R=60.2 \Omega$ led to one peak, $R=10.2 \Omega$ to two peaks, and $R=8.2 \Omega$ to four peaks (bottom row). These results confirm that the new oscillator presented in Fig. 1 is a robust stand-alone circuit.

Slight differences do exist between the PSpice attractors and the experimental ones. To the best of our knowledge, this can be explained by the fact that, experimentally, we measured the voltage at one end of any capacitor in relation to ground voltage, while with PSpice, it is the effective voltage difference at the two electrodes of any capacitor that is measured. On the other hand, real components include defects that are not always 
considered in the PSpice simulations. The comparison presented in Fig. 6 illustrates the good agreement between the three methods.

\section{CONCLUSION}

The challenge for finding the simplest systems and simplest circuits displaying chaos seems to involve an invariant: the simplest equations rarely correspond to the simplest circuits and vice versa. They seem to evolve in opposite directions. Piper and Sprott [27] have brought out a model matching a simple system with a simple circuit. In the same sense, we have proposed here a simple system, whose jerk function, although a bit complicated, resembles that of Chua's circuit aside from some parameters and has the sensible advantage to be governed by a PW linear equation that can be used to perform exact analytical works. Our circuit is nevertheless a remarkable jerk circuit, since it is based on a single Op-Amp as the only idealized component and carries a minimal number of five components, that is to say two components less than in the circuit of [27]. The numerical results presented match well the PSpice simulations of our laboratory experiment using real components.

\section{ACKNOWLEDGMENT}

R. Tchitnga would like to thank Pham et al. [19] for their remark on the term simplest in the title of one of his previous papers. This has inspired him and motivated the present work.

\section{REFERENCES}

[1] E. N. Lorenz, "Deterministic nonperiodic flow," J. Atmos. Sci., vol. 20, no. 2, pp. 130-141, Mar. 1963.

[2] O. E. Rössler, "Continuous chaos-Four prototype equations," Ann. New York Acad. Sci., vol. 316, pp. 376-392, Feb. 1979.

[3] H. A. Posch, W. G. Hoover, and F. J. Vesely, "Canonical dynamics of the Nosé oscillator: Stability, order, and chaos," Phys. Rev. A, At., Mol., Opt. Phys., vol. 33, no. 6, pp. 4253-4265, Jun. 1986.

[4] E. N. Lorenz, The Essence of Chaos. Seattle, WA, USA: Univ. of Washington Press, 1993, ch. 4, p. 148.

[5] J. C. Sprott, "Automatic generation of strange attractors," Comput. Graph., vol. 17, pp. 25-332, 1993.

[6] H. P. W. Gottlieb, "Question \#38. What is the simplest jerk function that gives chaos?" Amer. J. Phys., vol. 64, no. 5, p. 525, May 1996.

[7] S. H. Schot, "Jerk: The time rate of change of acceleration," Amer. J. Phys., vol. 46, no. 11, pp. 1090-1094, Nov. 1978.

[8] J. C. Sprott, "Some simple chaotic flows," Phys. Rev. E., Stat., Nonlinear, Soft Matter Phys., vol. 50, no. 2, pp. R647-R650, Aug. 1994.

[9] J. C. Sprott and S. J. Linz, "Algebraically simple chaotic flows," Int. J. Chaos Theory Appl., vol. 5, no. 2, pp. 1-20, 2000.

[10] J. C. Sprott, "Simple chaotic systems and circuits," Amer. J. Phys., vol. 68 , no. 8 , pp. $758-763$, Aug. 2000

[11] T. Matsumoto, L. O. Chua, and S. Tanaka, "Simplest chaotic nonautonomous circuit," Phys. Rev. A, At., Mol., Opt. Phys., vol. 30, no. 2, pp. 1155-7, Aug. 1984

[12] J. H. B. Deane, "Modeling the dynamics of nonlinear inductor circuits," IEEE Trans. Magn., vol. 30, no. 5, pp. 2795-2801, Sep. 1994.

[13] K. Murali, M. Laksmanan, and L. O. Chua, "The simplest dissipative nonautonomous chaotic circuit," IEEE Trans. Circuits Syst. I, Fundam. Theory Appl., vol. 41, no. 6, pp. 462-463, Jun. 1994.

[14] E. Lindberg, K. Murali, and A. Tamasevicius, "The smallest transistorbased nonautonomous chaotic circuit," IEEE Trans. Circuits Syst. II, Exp. Briefs, vol. 52, no. 10, pp. 661-664, Oct. 2005.

[15] L. O. Chua, "The genesis of Chua's circuit," Archiv Elektronik Uebertragungstechnik, vol. 46, no. 4, pp. 250-257, 1992.

[16] G.-S. Yim et al., "Chaotic behaviors of operational amplifiers," Phys. Rev. E., Stat., Nonlinear, Soft Matter Phys., vol. 69, no. 4, Apr. 2004, Art. ID. 045201

[17] B. Muthuswamy and L. O. Chua, "Simplest chaotic circuit," Int. J. Bifurcation Chaos, vol. 20, no. 5, pp. 1567-80, May 2010.
[18] B. Muthuswamy, A. Przybylski, C. Feilbach, and J. Mossbrucker, "Two element chaotic and hyperchaotic circuits," in Proc. 4th Int. Interdisc. Symp. Chaos Complex Syst., Antalya, Turkey, Apr. 30, 2012, pp. 1-10.

[19] V.-T. Pham, A. Buscarino, L. Fortuna, and M. Frasca, "Simple memristive time-delay chaotic systems," Int. J. Bifurcation Chaos, vol. 23, no. 4, Apr. 2013, Art. ID. 1350073.

[20] R. Tchitnga, H. B. Fotsin, B. Nana, P. H. Louodop Fotso, and P. Woafo, "Hartley's oscillator: The simplest chaotic two-component circuit," Chaos Solitons Fractals, vol. 45, no. 3, pp. 306-313, Mar. 2012.

[21] R. Tchitnga, R. Zebaze Nanfa'a, F. B. Pelap, P. H. Louodop Fotso, and P. Woafo, "A novel high-frequency interpretation of a general purpose Op-Amp-based negative resistance for chaotic vibrations in a simple a priori nonchaotic circuit," J. Vib. Control, to be published, DOI: $10.1177 / 1077546315585424$.

[22] J. G. Freire and J. A. C. Gallas, "Cyclic organization of stable periodic and chaotic pulsations in Hartley's oscillator," Chaos Solitons Fractals, vol. 59, pp. 129-134, Feb. 2014.

[23] A. S. Elwakil and A. M. Soliman, "Current conveyor chaos generators," IEEE Trans. Circuits Syst. I, Fundam. Theory Appl., vol. 46, no. 3, pp. 393-398, Mar. 1999.

[24] A. S. Elwakil and M. P. Kennedy, "A family of Colpitts-like chaotic oscillators," J. Franklin Inst., vol. 336, no. 4, pp. 687-700, May 1999.

[25] A. S. Elwakil and M. P. Kennedy, "A semi-systematic procedure for producing chaos from sinusoidal oscillators using diode-inductor and FET-capacitor composites," IEEE Trans. Circuits Syst. I, Fundam. Theory Appl., vol. 47, no. 4, pp. 582-590, Apr. 2000.

[26] A. S. Elwakil and M. P. Kennedy, "Construction of classes of circuitindependent chaotic oscillators using passive-only nonlinear devices," IEEE Trans. Circuits Syst. I, Fundam. Theory Appl., vol. 48, no. 3, pp. 289-307, Mar. 2001.

[27] J. R. Piper and J. C. Sprott, "Simple autonomous chaotic circuits," IEEE Trans. Circuits Syst. II, Exp. Briefs, vol. 57, no. 9, pp. 730-734, Sep. 2010.

[28] A. Sack, J. G. Freire, E. Lindberg, T. Pöschel, and J. A. C. Gallas, "Discontinuous spirals of stable periodic oscillations," Sci. Rep., vol. 3, p. 3350, 2013.

[29] T. Tsubone and T. Saito, "Stabilizing and destabilizing control for a piecewise-linear circuit," IEEE Trans. Circuits Syst. I, Fundam. Theory Appl., vol. 45, no. 2, pp. 172-177, Feb. 1998.

[30] J. C. Sprott, "A new chaotic jerk circuit," IEEE Trans. Circuits Syst. II, Exp. Briefs, vol. 58, no. 4, pp. 240-243, Apr. 2011.

[31] Z. Fu and J. Heidel, "Erratum: Non-chaotic behavior in three-dimensional quadratic systems," Nonlinearity, vol. 12, no. 3, p. 739, 1999.

[32] L. O. Chua, "Memristor-The missing circuit element," IEEE Trans. Circuit Theory, vol. CT-18, no. 5, pp. 507-519, Sep. 1971.

[33] D. B. Strukov, G. S. Snider, D. R. Stewart, and R. S. Williams, "The missing memristor found," Nature, vol. 453, no. 7191, pp. 80-83, May 2008.

[34] L. O. Chua, "The fourth element," Proc. IEEE, vol. 100, no. 6, pp. 1920-1927, Jun. 2012.

[35] S. Peotta and M. Di Ventra, "Superconducting memristors," Phys. Rev Appl., vol. 2, no. 3, Sep. 2014, Art. ID. 034011.

[36] F. P. Penfield, Jr., "Frequency Power Formulas for Josephson Junctions. Microwave and Millimeter Wave Techniques," MIT, Cambridge, MA, USA, MIT Tech. Rep. QPR-113, 1974.

[37] B. Muthuswamy et al., "Memristor modelling," in Proc. IEEE ISCAS, May 2014, pp. 490-493.

[38] L. O. Chua and C. Tseng, "A memristive circuit model for PN junction diodes," Int. J. Circuits Theory Appl., vol. 2, no. 4, pp. 367-389, Dec. 1974.

[39] M. P. Sah et al., "A generic model of memristors with parasitic components," IEEE Trans. Circuits Syst. I, Reg. Papers, vol. 62, no. 3, pp. 891-898, Mar. 2015.

[40] A. S. Elwakil and A. M. Soliman, "Two modified for chaos negative impedance converter Op Amp oscillators with symmetrical and antisymmetrical nonlinearities," Int. J. Bifurcation Chaos, vol. 8, no. 6, pp. 1335-1346, Jun. 1998

[41] J. L. Hudgins, T. F. Bogart, Jr., K. Mayaram, M. P. Kennedy, and G. Kolumban, Nonlinear Circuits, the Electrical Engineering Handbook, R. C. Dorf, Ed. Boca Raton, FL, USA: CRC Press, 2000.

[42] T. F. Bogart, Jr., Electronic Devices and Circuits, 3rd ed. Columbus, OH, USA: Macmillan/Merrill, 1993, p. 694.

[43] J. C. Sprott, Chaos and Time-Series Analysis. New York, NY, USA: Oxford Univ. Press, 2003.

[44] M. Ferraro, N. Furman, Y. Liu, M. C. Mariani, and D. Rial, "Analysis of intermittence, scale invariance and characteristic scales in the behavior of major indices near a crash," Phys. A, Stat. Mech. Appl., vol. 359, pp. 576-588, Jan. 2006. 